\title{
THE LAGRANGE INTERPOLATION FORMULA AND STIRLING NUMBERS
}

H. W. GOULD

1. Nörlund $\left[4\right.$, p. 146] has defined the polynomial $B_{n}^{(2)}$ by means of

$$
\left(\frac{x}{e^{x}-1}\right)^{z}=\sum_{n=0}^{\infty} B_{n}^{(z)} \frac{x^{n}}{n !} \text {. }
$$

Thus $B_{n}^{(z)}$ is a polynomial in $z$ of degree $n$.

The Stirling numbers $S_{1}(n, k)$ and $S_{2}(n, k)$ of the first and second kind, respectively, are related to Nörlund's polynomial by means of

$$
\begin{aligned}
(-1)^{k} S_{1}(n-1, k) & =\left(\begin{array}{c}
n-1 \\
k
\end{array}\right) B_{k}^{(n)}, \\
S_{2}(n, k) & =\left(\begin{array}{c}
n+k \\
k
\end{array}\right) B_{k}^{(-n)},
\end{aligned}
$$

and the formulas may be used to extend the definition of $S_{1}(n, k)$ and $S_{2}(n, k)$ for arbitrary real $n$.

In a previous paper [2] the writer has proved several apparently new formulas relating the two kinds of Stirling numbers to each other. Carlitz [1] has generalized these results in part as follows. Instead of considering the polynomial $B_{k}^{(z)}$, let $f_{k}(z)$ denote an arbitrary polynomial in $z$ of degree $k$, that is

$$
f_{k}(z)=\sum_{i=0}^{k} a_{i} z^{i}
$$

and let, moreover,

$$
f_{k}(0)=0 \quad(k \geqq 1) .
$$

In addition if we define

$$
\begin{aligned}
(-1)^{k} F_{1}(n-1, k) & =\left(\begin{array}{c}
n-1 \\
k
\end{array}\right) f_{k}(n), \\
F_{2}(n, k) & =\left(\begin{array}{c}
n+k \\
k
\end{array}\right) f_{k}(-n),
\end{aligned}
$$

then

Received by the editors August 29, 1959. 


$$
\begin{aligned}
& F_{1}(n-1, k)=\sum_{j=0}^{k}\left(\begin{array}{l}
k-n \\
k+j
\end{array}\right)\left(\begin{array}{l}
k+n \\
k-j
\end{array}\right) F_{2}(j, n), \\
& F_{2}(n-k, k)=\sum_{j=0}^{k}\left(\begin{array}{l}
k-n \\
k+j
\end{array}\right)\left(\begin{array}{l}
k+n \\
k-j
\end{array}\right) F_{1}(k+j-1, k) .
\end{aligned}
$$

Carlitz proved these using only elementary properties of polynomials.

In the writer's paper [2], the proofs of the original formulas when $F_{1}$ and $F_{2}$ are the Stirling numbers was made to depend upon an application of the generalized chain rule of differentiation in the form

$$
z^{n} D_{x}^{k} z^{-n}=\sum_{j=0}^{k}\left(\begin{array}{c}
-n \\
j
\end{array}\right)\left(\begin{array}{c}
k+n \\
k-j
\end{array}\right) z^{-j} D_{x}^{k} z^{j}
$$

and a function like that in relation (1).

2. It may be of interest to point out that relations (8), (9) and (10) are also obtainable from the Lagrange interpolation formula in a rather easy manner.

The Lagrange interpolation formula may be written in the form

$$
f_{k}(u)=\sum_{j=0}^{k} f_{k}\left(u_{j}\right) \prod_{i=0 ; i \neq j}^{k} \frac{u-u_{i}}{u_{j}-u_{i}}
$$

and is an algebraic identity when $f_{k}(u)$ is a polynomial in $u$ of degree $k$.

We first note that

$$
\prod_{i=0 ; i \neq j}^{k}(j-i)=\frac{(-1)^{k-i} k !}{\left(\begin{array}{l}
k \\
j
\end{array}\right)} \quad(0 \leqq j \leqq k, k \geqq 1),
$$

from which it follows that

$$
\left(\begin{array}{c}
-n \\
j
\end{array}\right)\left(\begin{array}{c}
k+n \\
k-j
\end{array}\right)=\prod_{i=0 ; i \neq j}^{k} \frac{-n-i}{j-i} \quad(0 \leqq j \leqq k, n \text { real }) .
$$

Next we note the binomial coefficient identity

$$
\left(\begin{array}{c}
-n \\
j
\end{array}\right)\left(\begin{array}{c}
n-1 \\
k
\end{array}\right)=(-1)^{k}\left(\begin{array}{c}
k-n \\
k+j
\end{array}\right)\left(\begin{array}{c}
k+j \\
j
\end{array}\right) \text {. }
$$

It follows from (13) and (14) that

$$
\left(\begin{array}{c}
k-n \\
k+j
\end{array}\right)\left(\begin{array}{c}
k+n \\
k-j
\end{array}\right)=(-1)^{k}\left(\begin{array}{c}
n-1 \\
k
\end{array}\right)\left(\begin{array}{c}
k+j \\
k
\end{array}\right)^{-1} \prod_{i=0 ; i \neq j}^{k} \frac{-n-\imath}{j-i}
$$


so that relation (8) becomes

$$
\begin{aligned}
& \left(\begin{array}{c}
n-1 \\
k
\end{array}\right)^{-1} F_{1}(n-1, k) \\
& \qquad=\sum_{j=0}^{k}(-1)^{k}\left(\begin{array}{c}
k+j \\
k
\end{array}\right)^{-1} F_{2}(j, k) \prod_{i=0 ; i \neq j}^{k} \frac{-n-i}{j-i}
\end{aligned}
$$

or, in view of (6) and (7),

$$
f_{k}(-n)=\sum_{j=0}^{k} f_{k}(-j) \prod_{i=0 ; i \neq j}^{k} \frac{-n-i}{j-i},
$$

which obviously is a special case of the Lagrange formula (11) applied in the case of the polynomial $f_{k}(u)$ with $u=-n$ and $u_{j}=j$.

Similar remarks apply to relation (9).

3. To show that (10) is also derivable from (11) we need to show that we may choose

$$
f_{k}(u)=z^{u} D_{x}^{k} z^{-u}
$$

With this as a definition of $f_{k}(u)$ we must show that $f_{k}(u)$ is a polynomial in $u$ of degree $k$, that is

$$
f_{k}(u)=\sum_{i=0}^{k} a_{i}^{k}(x, z) u^{i},
$$

where the coefficients $a_{i}^{k}(x, z)$ are independent of $u$. We remark that (18) yields $f_{k}(0)=0 \quad(k \geqq 1)$ which is the hypothesis (5) made by Carlitz.

Clearly $f_{0}(u)=1$ and

$$
f_{1}(u)=-u z^{-1} D_{x} z
$$

It will be sufficient for an inductive proof of (19) then to examine $f_{k+1}(u)$ and show that the new coefficients arising in it are of the same type as those in $f_{k}(u)$.

Now a fundamental and easily verified property of the generalized chain rule (see for example $[3$, p. 21] for a detailed proof) is that

$$
D_{x}^{k} z^{-u}=\sum_{j=0}^{k} A_{j}^{k}(x) \cdot D_{z}^{j} z^{-u}
$$

where $u$ is independent of $x$ and $z, z=z(x)$, and the coefficients $A_{j}^{k}(x)$ are polynomials in the derivatives of order $\leqq k$ of $z(x)$. As a matter of fact this follows from the recurrence relation 


$$
A_{j}^{k+1}(x)=A_{j-1}^{k}(x) \cdot D_{x} z+D_{x} A_{j}^{k}(x),
$$

together with the conditions that

$$
A_{j}^{k}(x)=0 \text { if either } j<0 \text { or } j>k .
$$

From (21) and (18) it follows that

$$
f_{k}(u)=\sum_{j=0}^{k} A_{j}^{k}(x) \cdot z^{u} D_{z}^{j} z^{-u}
$$

and it will therefore be sufficient for our purposes to show that

$$
z^{u} D_{z}^{j} z^{-u}=\sum_{i=0}^{j} b_{i}^{j}(z) u^{i}
$$

where the $b_{i}^{j}(z)$ are independent of $u$. Indeed we have

$$
\begin{aligned}
z^{u} D_{z}^{j+1} z^{-u} & =z^{u} D_{z^{-u}} z^{-u}\left(z^{u} D_{z^{j}}^{j-u}\right) \\
& =z^{u} D_{z} z^{-u} \sum_{i=0}^{j} b_{i}^{j}(z) u^{i} \\
& =\sum_{i=0}^{j} u^{i} z^{u}\left[-u z^{-u-1} b_{i}^{j}(z)+z^{-u} D_{z} b_{i}^{j}(z)\right] \\
& =\sum_{i=0}^{j+1} u^{i}\left[-z^{-1} b_{i-1}^{j}(z)+D_{z} b_{i}^{j}(z)\right] .
\end{aligned}
$$

Consequently if we assume the recurrence formula

$$
b_{i}^{j+1}(z)=-z^{-1} b_{i-1}^{j}(z)+D_{z} b_{i}^{j}(z)
$$

together with the conditions that $b_{i}^{j}(z)=0$ if either $i<0$ or $i>j$, then clearly this implies that $b_{i}^{j}(z)$ can only involve powers of $z$ and will not depend on $u$ so that (19) is proved.

Therefore the Lagrange formula (11) may be applied to the polynomial function defined by (19), and with the choice $u=n$ and $u_{j}=j$ we find that

$$
z^{n} D_{x}^{k} z^{-n}=\sum_{j=0}^{k} z^{-j} D_{x z}^{k} \prod_{i=0 ; i \neq j}^{k} \frac{n-i}{j-i}
$$

\section{REFERENCES}

1. L. Carlitz, Note on Nörlund's polynomial $B_{n}^{(s)}$, Proc. Amer. Math. Soc. vol. 11 (1960) pp. 452-455.

2. H. W. Gould, Stirling number representation problems, Proc. Amer. Math. Soc. vol. 11 (1960), pp. 447-451. 
3. - The Stirling numbers, University of Virginia, Master's thesis 2130, June, 1956.

4. N. E. Nörlund, Vorlesungen über Differenzenrechnung, Berlin, 1924.

WEST VIRGINIA UNIVERSITY

\title{
SARIO'S LEMMA ON HARMONIC FUNCTIONS ${ }^{1}$
}

\author{
KÔTARO OIKAWA
}

1. The alternating method of constructing a harmonic function on a Riemann surface due to $H$. A. Schwarz is discussed from a general point of view by Sario [2]. To show the convergence of the Neumann series, he uses the following lemma ([2, p. 282]; it has another expression but is evidently equivalent to the following):

SARIo's Lemma. Let $W$ be an open Riemann surface and $K$ be a compact set on $W$. Then there exists a constant $q$ such that $0<q<1$ and that

$$
\max _{K}|u| \leqq q \cdot \sup _{W}|u|
$$

for every harmonic function $u$ which changes sign on $K$.

The present note is devoted to evaluating the constant $q$ according to oral suggestions of Professor S. Warschawski, to whom the author wishes to express his heartiest gratitude. We are of course interested in finding the smallest possible value. However, we have succeeded in doing so only for a very special case.

2. It is well known that we can introduce the Poincaré metric

$$
d s=\frac{|d z|}{1-|z|^{2}}
$$

in the unit disc $|z|<1$. It is invariant under linear transformations of $|z|<1$ onto itself.

For $z_{1}$ and $z_{2}$, the infimum of $\int_{C} d s$ with respect to all the curves $C$ connecting $z_{1}$ with $z_{2}$ will be denoted by $d\left(z_{1}, z_{2}\right)$. For instance,

$$
d(0, z)=\frac{1}{2} \log \frac{1+|z|}{1-|z|} .
$$

The geodesic connecting $z_{1}$ with $z_{2}$ is the arc contained in the circle perpendicular to $|z|=1$ (see, e.g., [1]).

Received by the editors June 8, 1959 and, in revised form, July 27, 1959.

1 This paper was prepared under Contract No. DA-04-495-ORD-722, OOR Project No. 1517 between the University of California, Los Angeles and the Office of Ordnance Research, U. S. Army. 